Korean J. Math. 19 (2011), No. 2, pp. 233-242

\title{
STUDY ON THE PERTURBED PIECEWISE LINEAR SUSPENSION BRIDGE EQUATION WITH VARIABLE COEFFICIENT
}

\author{
Tacksun Jung And Q-Heung ChOI*
}

\begin{abstract}
We get a theorem that there exist at least two solutions for the piecewise linear suspension bridge equation with variable coefficient jumping nonlinearity and Dirichlet boundary condition when the variable coefficient of the nonlinear term crosses first two successive negative eigenvalues. We obtain this multiplicity result by applying Leray-Schauder degree theory.
\end{abstract}

\section{Introduction and statement of main result}

The suspension bridge equation is considered as a model of the nonlinear oscillations in differential equation. We consider a one-dimensional beam of length $\pi$ suspended by cables. When the cables are stretched, there is a restoring force which is assumed to be proportional to the amount of the stretching. But when the beam moves in the opposite direction, then there is no restoring force exerted on it. If $u(x, t)$ denotes the displacement in the downward direction at position $x$ and time $t$, then a simplified model is given by the equations.

$$
\begin{gathered}
u_{t t}+K_{1} u_{x x x x}+K_{2} u^{+}=W(x)+\epsilon f(x, t), \\
u(0, t)=u(L, t)=0, \quad u_{x x}(0, t)=u_{x x}(L, t)=0,
\end{gathered}
$$

where $u^{+}=\max \{0, u\}$. Let $b(x)$ be a Hölder continuous function. In this paper we investigate the multiplicity of the piecewise linear suspension bridge equation with variable coefficient and Dirichlet boundary

Received May 19, 2011. Revised June 7, 2011. Accepted June 10, 2011.

2000 Mathematics Subject Classification: 35Q72.

Key words and phrases: Suspension bridge equation, Leray-Schauder degree theory.

This work(Choi) was supported by Inha University Research Grant.

${ }^{*}$ Corresponding author. 
condition

$$
\begin{aligned}
u_{t t}+u_{x x x x}+b(x) u^{+} & =1+\epsilon h(x, t) \quad \text { in }\left[-\frac{\pi}{2}, \frac{\pi}{2}\right] \times R, \\
u\left( \pm \frac{\pi}{2}, t\right) & =u_{x x}\left( \pm \frac{\pi}{2}, t\right)=0
\end{aligned}
$$

$u$ is $\pi$ - periodic in $t$ and even in $x$ and $t$,

where $u^{+}=\max \{0, u\}$. McKenna and Walter [3] proved that if $b(x)=$ $b$ constant and $3<b<15$, then (1.1) with (1.2) and (1.3) has at least two solutions by degree theory. Choi and Jung [1] also proved that if $3<b<15$, then (1.1) with (1.2) and (1.3) has at least three solutions by the variational reduction method, with replacing the condition for $u(t, x)$ in (1.3) by

$$
u \text { is } \pi \text {-periodic in } t \text { and even in } x \text {. }
$$

Micheletti and Saccon [4] proved that there exists a number $\delta_{k}>0$ such that for any $b$ with $\Lambda_{k}^{-}-\delta_{k}<-b<\Lambda_{k}^{-}$and $\Lambda_{k}^{-}<\Lambda_{1}^{-}$(1.1) with free-ends boundary conditions, and replacing the right hand side of (1.1) by $c>0$ has at least four nontrivial solutions via the critical point theory on the manifold with boundary induced from the limit relative category of the torus with one hole. In this paper we improve these results: We prove that when the variable coefficient of the piecewise linear part crosses first two successive two negative eigenvalues, (1.1) with (1.2) and (1.3) has at least two nontrivial solutions.

To state main result explicitly we need the following notations:

The eigenvalue problem

$$
u_{t t}+u_{x x x x}=\lambda u
$$

with (1.2) and (1.3) has infinitely many eigenvalues

$$
\lambda_{m n}=(2 n+1)^{4}-4 m^{2} \quad(m, n=0,1,2, \ldots)
$$

and corresponding normalized eigenfunctions $\phi_{m n}(m, n \geq 0)$ given by

$$
\begin{gathered}
\phi_{0 n}=\frac{\sqrt{2}}{\pi} \cos (2 n+1) x \quad \text { for } n \geq 0, \\
\phi_{m n}=\frac{2}{\pi} \cos 2 m t \cos (2 n+1) x \quad \text { for } m>0, n \geq 0 .
\end{gathered}
$$

We note that all eigenvalues in the interval $(-19,45)$ are given by

$$
\lambda_{20}=-15<\lambda_{10}=-3<\lambda_{00}=1<\lambda_{41}=17 .
$$


Let $H$ be the space introduced in section 2 .

Theorem 1.1. Let $h \in H,\|h\|=1$ and $b(x)$ is a Hölder continuous function with $-15<-b(x)<-3$. Then there exists $\epsilon_{0}>0$ depending on $h$ and $b$ such that if $|\epsilon|<\epsilon_{0}$, problem (1.1) with (1.2) and (1.3) has at least two nontrivial solutions.

The outline of the proof is as follows: In section 2, we investigate a priori bound for the solutions and the equilibrium solution of (1.1) with (1.2) and (1.3). In section 3, we prove Theorem 1.1 by applying the Leray-Schauder degree theory.

\section{A priori bound and the equilibrium solution} by

Let $Q$ be the square $\left[-\frac{\pi}{2}, \frac{\pi}{2}\right] \times\left[-\frac{\pi}{2}, \frac{\pi}{2}\right]$ and $H_{0}$ the Hilbert space defined

$$
H_{0}=\left\{u \in L^{2}(Q) \mid u \text { is even in } x\right\} .
$$

The set of functions $\left\{\phi_{m n}\right\}$ is an orthonormal base in $H_{0}$. We define a subspace $H$ of $H_{0}$ as follows

$$
H=\left\{u \in H_{0}\left|u=\sum h_{m n} \phi_{m n}, \quad \sum\right| \lambda_{m n} \mid h_{m n}^{2}<\infty\right\}
$$

with a norm

$$
\|u\|=\left[\sum\left|\lambda_{m n}\right| h_{m n}^{2}\right]^{\frac{1}{2}} .
$$

Then this normed space $H$ is complete. We are looking for weak solutions of (1.1) with ( 1.2) and (1.3). A weak solution of (1.1) with (1.2) and (1.3), which is also called a solution in $H$, is of the form

$$
u=\sum c_{m n} \phi_{m n} \quad \text { with } u_{t t}+u_{x x x x}=\sum \lambda_{m n} c_{m n} \phi_{m n} \in H,
$$

i.e., with $\sum c_{m n}^{2} \lambda_{m n}^{2}<\infty$, which implies $u \in H$. Thus a weak solution of (1.1) with (1.2) and (1.3) is characterized by

$$
u_{t t}+u_{x x x x}+b(x) u^{+}=1+\epsilon h(x, t) \quad \text { in } H .
$$

Now we consider the eigenvalue problem

$$
\begin{aligned}
y_{x x x x}+b(x) y & =\Lambda_{n} y \quad \text { in }\left(-\frac{\pi}{2}, \frac{\pi}{2}\right), \\
y\left( \pm \frac{\pi}{2}\right) & =y^{\prime \prime}\left( \pm \frac{\pi}{2}\right)=0 .
\end{aligned}
$$

McKenna and Walter [2] showed that (2.2) has infinitely many eigenvalues $\Lambda_{n}, n \geq 1$, and the corresponding eigenfunctions $\psi_{n}, n \geq 1$. We 
assume that the eigenfunctions are normalized with respect to $H$ inner product (the space $H$ is introduced in section 2). Standard eigenvalue theory gives that

$$
\begin{gathered}
\Lambda_{0}<\Lambda_{1} \leq \Lambda_{2} \leq \cdots, \quad \Lambda_{k} \rightarrow+\infty \quad \text { as } k \rightarrow+\infty, \\
\psi_{0}(x)>0 \quad \text { in } \Omega .
\end{gathered}
$$

We have some propositions which are proved in [1].

Lemma 2.1. (i) $u_{t t}+u_{x x x x} \in H$ implies $u \in H$.

(ii) $\|u\| \geq\|u\|_{L^{2}}$, where $\|u\|_{L^{2}}$ denotes the $L^{2}$ norm of $u$.

(iii) $\|u\|=0$ iff $\|u\|_{L^{2}}=0$.

Lemma 2.2. Let $h \in H$ with $\|h\|=1$ and $\alpha>0$ be given. Then there exists a constant $s_{0}>0$ so large enough that for all $s \geq s_{0}$, all $b(x)$ with $-15+\alpha \leq-b(x) \leq 1-\alpha$ and all $\epsilon \in[-1,1]$, the problem

$$
u_{t t}+u_{x x x x}+b(x) u^{+}=1+\epsilon h(x, t)+s \psi_{0}(x) \text { in } H,
$$

has no solution.

Proof. We suppose that the lemma is false. Then there exist a sequence $\left(b_{n}(x), \epsilon_{n}, u_{n}\right)$ with $b_{n}(x) \in[-15+\alpha, 1-\alpha],\left|\epsilon_{n}\right| \leq 1, u_{n}$ in $H$ and $\left(s_{n}\right)_{n \in N}$ such that $\lim _{n \in \infty} s_{n} \rightarrow+\infty$ and $u_{n}$ are the solutions of

$$
u_{n}=\left(D_{t t}+D_{x x x x}\right)^{-1}\left(-b_{n}(x) u_{n}^{+}+1+\epsilon_{n} h+s_{n} \psi_{0}(x)\right), \quad \text { in } H
$$

We claim that $\left\{u_{n}\right\}$ is unbounded. In fact, if $\left\{u_{n}\right\}$ is bounded, then $\lim _{n \rightarrow \infty} \frac{u_{n}}{s_{n}}=0$ in $Q$, strongly in $L^{2}(Q)$ and weakly in $H$. Dividing (2.3) by $s_{n}$, we have

$$
\frac{u_{n}}{s_{n}}=\left(D_{t t}+D_{x x x x}\right)^{-1}\left(-b_{n}(x)\left(\frac{u_{n}}{s_{n}}\right)^{+}+\frac{1+\epsilon_{n} h}{s_{n}}+\psi_{0}(x)\right) .
$$

Passing to the limit to both sides of (2.4), we have that

$$
0=\left(D_{t t}+D_{x x x x}\right)^{-1}\left(\psi_{0}(x)\right),
$$

which is a contradiction because the left hand side of the equality is 0 , but the right hand side is not equal to 0 . Thus $\lim _{n \rightarrow \infty}\left\|u_{n}\right\|=\infty$. Let $z_{n}=\frac{u_{n}}{\left\|u_{n}\right\|}$. Since $\left\{z_{n}\right\}$ is compact, there exists a subsequence, up to a subsequence, $\left\{z_{n}\right\}$ such that $\lim _{n \in N} z_{n}=z$ a.e. in $Q$, strongly in $L^{2}(Q)$ and weakly in $H$. Dividing (2.3) by $\left\|u_{n}\right\|$, we have

$$
\left.z_{n}=\left(D_{t t}+D_{x x x x}\right)^{-1}\left(-b_{n}(x) z_{n}^{+}+\frac{1+\epsilon_{n} h}{\left\|u_{n}\right\|}\right)+\frac{s_{n}}{\left\|u_{n}\right\|} \psi_{0}(x)\right) .
$$


We suppose that $\lim _{n \rightarrow \infty} \frac{s_{n}}{\left\|u_{n}\right\|}=s \geq 0$. Passing to the limit in (2.5), we obtain that

$$
z=\left(D_{t t}+D_{x x x x}\right)^{-1}\left(-b(x) z^{+}+s \psi_{0}(x)\right) .
$$

We claim that (2.6) has only trivial solution $z=0$. In fact, we suppose that $z \neq 0$. Multiplying $\psi_{0}(x)$ to both sides of (2.6) and integrating, we have

$$
\begin{aligned}
0 & \leq s=\int_{\Omega}\left(\left(D_{t t}+D_{x x x x}\right) z \psi_{0}(x)+b(x) z^{+} \psi_{0}(x)\right) d x \\
& =\int_{\Omega}\left(\left(D_{t t}+D_{x x x x}+b(x)\right) \psi_{0}(x) z^{+}-\left(D_{t t}+D_{x x x x}\right) \psi_{0}(x) z^{-}\right) d x
\end{aligned}
$$

which is absurd because the right hand side of the above equation is negative since $\Lambda_{0}<0$ and $-\left(\left(D_{t t}+D_{x x x x}\right) \psi_{0}(x) z^{-}=-D_{x x x x} \psi_{0}(x) z^{-}<\right.$ 0 . Thus $z=0$. This is a contradiction since $\|z\|=1$. We prove the lemma.

The next lemma established a priori bounds for the solutions of (2.2).

Lemma 2.3. (a priori bound) Let $h \in H$ with $\|h\|=1$ and $\alpha>0$ be given. Then there exist a constant $C>0$ and $s_{*}>0$ with $s_{*}<s_{0}$ such that for all $s \leq s_{*}$, all $b(x)$ with $-15+\alpha \leq-b(x) \leq 1-\alpha$ and all epsilon $\in[-1,1]$, the solutions $u$ of (2.2) satisfy $\|u\| \leq C$.

Proof. By contradiction, we suppose that there exists a sequence $\left(b_{n}(x), \epsilon_{n}, u_{n}\right)$ with $b_{n}(x) \in[-15+\alpha, 1-\alpha],\left|\epsilon_{n}\right| \leq 1, u_{n}$ in $H$ and $\left(s_{n}\right)_{n \in N}$ such that $\left\|u_{n}\right\| \rightarrow \infty, b_{n}(x) \rightarrow b(x) \in[-15+\alpha, 1-\alpha], s_{n} \rightarrow s_{*}$, $s_{n} \leq s_{*}$ and $u_{n}$ satisfy the equation

$$
\begin{aligned}
& \left(D_{t t}+D_{x x x x}+b_{n}(x)-\Lambda_{0}\right) u_{n} \\
& \quad=1+\epsilon_{n} h(x, t)+\left(b_{n}(x)-\Lambda_{0}\right) u_{n}^{+}-\left(b_{n}(x)-\Lambda_{0}\right) u_{n}^{-}+s_{n} \psi_{0}(x) .
\end{aligned}
$$

Let $z_{n}=\frac{u_{n}}{\left\|u_{n}\right\|}$. By the compactness of $\left\{z_{n}\right\}$, there exists $z$ such that $z_{n} \rightarrow z$, and $z$ is a solution of the equation

$$
\left(D_{t t}+D_{x x x x}+b(x)-\Lambda_{0}\right) z=-\Lambda_{0} z^{+}+\Lambda_{0} z^{-}-b(x) z^{-} .
$$

Taking inner product of both sides of (2.8) with $\psi_{0}(x)$, we have

$$
\begin{aligned}
0 & =\left(\left(D_{t t}+D_{x x x x}+b(x)-\Lambda_{0}, \psi_{0}(x)\right)\right. \\
& =\left(-\Lambda_{0} z^{+}+\left(\Lambda_{0}-b(x)\right) z^{-}, \psi_{0}(x)\right) .
\end{aligned}
$$


Since $-\Lambda_{0} z^{+}+\left(\Lambda_{0}-b(x)\right) z^{-} \geq \epsilon\|z\|$, the right hand side of (2.9) is bigger than or equal to 0 . Thus the only possibility to hold (2.9) is that $z=0$, which is impossible since $\|z\|=1$. Thus we prove the lemma.

Lemma 2.4. Let $h \in H,\|h\|=1$ and $-15+\alpha \leq-b(x) \leq 1-\alpha$. Then there exists a constant $R>0$ (depending on $C$ which is introduced in Lemma 2.3) such that any solutions of (2.1) is contained in $B_{R}(0)$ and the Leray-Schauder degree

$$
d_{L S}\left(u-\left(D_{t t}+D_{x x x x}\right)^{-1}\left(-b(x) u^{+}+1+\epsilon h+s \psi_{0}(x)\right), B_{R}(0), 0\right)=0
$$

for $R>C$ and $s \leq s_{*}$.

Proof. By Lemma 2.2, there exists a constant $s_{0}>0$ such that if $s \geq s_{0},(2.2)$ has no solution. By Lemma 2.3, there exist a constant $C$ and $s_{*}>0$ with $s_{*}<s_{0}$ such that if $u$ is a solution of (2.2) with $s<s_{*}$, then $\|u\| \leq C$. Let us choose $R$ so large that $R>C$. We note that $u-\left(D_{t t}+D_{x x x x}\right)^{-1}\left(-b(x) u^{+}+1+\epsilon h+(1-\lambda) s_{0} \psi_{0}(x)\right) \neq 0$ $u-\left(D_{t t}+D_{x x x x}+b(x)-\Lambda_{0}\right)^{-1}\left(-\Lambda_{0} u^{+}+\left(\Lambda_{0}-b(x)\right) u^{-}+(1-\lambda) s_{0} \psi_{0}(x)\right)$ on $\partial B_{R}(0)$ for $0 \leq \lambda \leq 1$. By the homotopy invariance property, we have that the Leray-Schauder degree

$$
\begin{aligned}
& d_{L S}\left(u-\left(D_{t t}+D_{x x x x}\right)^{-1}\left(-b(x) u^{+}+1+\epsilon h+s \psi_{0}(x)\right), B_{R}(0), 0\right) \\
& =d_{L S}\left(u-\left(D_{t t}+D_{x x x x}\right)^{-1}\left(-b(x) u^{+}+1+\epsilon h\right.\right. \\
& \left.\left.\quad+(1-\lambda) s_{0} \psi_{0}(x)\right)+\lambda s \psi_{0}(x), B_{R}(0), 0\right) \\
& =d_{L S}\left(u-\left(D_{t t}+D_{x x x x}\right)^{-1}\left(-b(x) u^{+}+1+\epsilon h+s_{0} \psi_{0}(x)\right), B_{R}(0), 0\right) \\
& =0,
\end{aligned}
$$

where $0 \leq \lambda \leq 1$. Thus we prove the lemma.

REMARK 1. From Lemma 2.4

$$
d_{L S}\left(u-\left(D_{t t}+D_{x x x x}\right)^{-1}\left(-b(x) u^{+}+1+\epsilon h\right), B_{R}(0), 0\right)=0
$$

because $0<s_{*}$.

Lemma 2.5. For $-b(x)<1$ the linear boundary value problem

$$
\begin{gathered}
y_{x x x x}+b(x) y=1 \quad \text { in }\left(-\frac{\pi}{2}, \frac{\pi}{2}\right), \\
y\left( \pm \frac{\pi}{2}\right)=y^{\prime \prime}\left( \pm \frac{\pi}{2}\right)=0, \\
y \text { is even in } x
\end{gathered}
$$

has a unique positive solution $y$. 
Proof. (2.10) can be rewritten as

$$
\left(D_{x x x x}-(-b(x))\right) y=1
$$

with boundary condition. The operator $D_{x x x x}-(-b(x))$ is positive operator since the eigenvalues of the eigenvalue problem $\left(D_{x x x x}-(-b(x))\right) y=$ $\lambda y$ with boundary condition has are all $>0$. Since the right hand side of (2.11) is 1 and positive, the unique solution of (2.11) is positive.

Lemma 2.6. For $-b(x)<1$ the boundary value problem

$$
\begin{gathered}
y_{x x x x}+b(x) y^{+}=1 \quad \text { in }\left(-\frac{\pi}{2}, \frac{\pi}{2}\right), \\
y\left( \pm \frac{\pi}{2}\right)=y^{\prime \prime}\left( \pm \frac{\pi}{2}\right)=0 \\
y \text { is even in } x
\end{gathered}
$$

has a positive solution $y$.

Proof. The unique positive solution $y$ of (2.10) with boundary condition is also a solution of (2.12).

Lemma 2.7. Let $h \in H,\|h\|=1$ and $-15<-b(x)<-3$. Then there exists a constant $\eta>0, \epsilon_{0}>0$ such that the Leray-Schauder degree

$$
d_{L S}\left(u-\left(D_{t t}+D_{x x x x}\right)^{-1}\left(-b(x) u^{+}+1+\epsilon h\right), B_{\eta}(y), 0\right)=-1
$$

for $|\epsilon|<\epsilon_{0}$, where $y$ is the positive solution of (2.12)

Proof. (1.1) can rewritten as

$$
\left(D_{t t}+D_{x x x x}+b(x)\right) u=-b(x) u^{-}+1+\epsilon h(x, t) .
$$

or

$$
u=\left(D_{t t}+D_{x x x x}+b(x)\right)^{-1}\left(-b(x) u^{-}+1+\epsilon h(x, t)\right) .
$$

Let $K$ be the closure of $\left(D_{t t}+D_{x x x x}+b(x)\right)^{-1}(\bar{B})$, where $\bar{B}$ is the closed unit ball centered at $y$ in $L_{2}(\Omega)$. Let $u$ be a nontrivial solution of (2.1). Let $u=y+v$ and $\|v\|=\eta$. Then $v$ satisfies the equation

$$
\left(D_{t t}+D_{x x x x}+b(x)\right) v=-b(x)(y+v)^{-}+\epsilon h(x, t)
$$

or

$$
v=\left(D_{t t}+D_{x x x x}+b(x)\right)^{-1}\left(-b(x)(y+v)^{-}+\epsilon h(x, t)\right) .
$$

Let us set $\beta=\max |b(x)|$. Since $\left\|(y+v)^{-}\right\|<\left\|v^{-}\right\| \leq\|v\|$ and

$$
\left\|-b(x)(y+v)^{-}+\epsilon h(x, t)\right\| \leq \beta\|v\|+\epsilon .
$$


It follows that

$$
v \in\left(\epsilon_{0}+\beta \eta\right) K \text { for }|\epsilon|<\epsilon_{0} .
$$

Since $w \in K$ satisfies $\left\|(\eta w-y)^{+}\right\| \leq \eta \delta(\eta)$, we get from (2.16)

$$
\left\|(v+y)^{-}\right\|=\left\|(-v-y)^{+}\right\| \leq\left(\epsilon_{0}+\beta \eta\right) \delta\left(\epsilon_{0}+\beta \eta\right) .
$$

We set that $\left\|\left(D_{t t}+D_{x x x x}+b(x)\right)^{-1}\right\|=\frac{1}{\min \{-3+b(x),-b(x)+15\}}=L$. We choose $\epsilon_{0}$ such that $\epsilon_{0} \leq \frac{1}{2 L} \eta$. Then the norm of the right hand side of $(2.15)$ is

$$
\left\|\left(D_{t t}+D_{x x x x}+b(x)\right)^{-1}\left(-b(x)(y+v)^{-}+\epsilon h(x, t)\right)\right\| \leq \frac{1}{2} \eta+L\left(\frac{1}{2 L} \eta+b \eta\right) \delta(e t a+b \eta) .
$$

We choose $\eta>0$ so small that

$$
\left\|\left(D_{t t}+D_{x x x x}+b(x)\right)^{-1}\left(-b(x)(y+v)^{-}+\epsilon h(x, t)\right)\right\|<\eta .
$$

It follows that for this value of $\eta$ there is no solution of (2.1) of the form $u=y+v$ with $\|v\|=\eta$. The same conclusion holds for solutions $u=y+v$ of the equation

$$
\left(D_{t t}+D_{x x x x}+b(x)\right) u=1+\lambda\left(-b(x) u^{-}+\epsilon h(x, t)\right) .
$$

or

$$
u=\left(D_{t t}+D_{x x x x}+b(x)\right)^{-1}\left(1+\lambda\left(-b(x) u^{-}+\epsilon h(x, t)\right)\right),
$$

where $0 \leq \lambda \leq 1$. When $\lambda=1$, (2.17) gives the equation (2.1). It follows from (2.17) that

$$
v=\left(D_{t t}+D_{x x x x}+b(x)\right)^{-1}\left(\lambda\left(-b(x)(y+v)^{-}+\epsilon h(x, t)\right)\right) .
$$

Now we have the same conclusion for the equation (2.18). That is, there is no solution $u=y+v$ with $\|v\|=\eta$. Since the Leray-Schauder degree is invariant under a homotopy, we have

$$
\begin{aligned}
& d_{L S}\left(u-\left(D_{t t}+D_{x x x x}\right)^{-1}\left(-b(x) u^{+}+1+\epsilon h\right), B_{\eta}(y), 0\right) \\
& =d_{L S}\left(u-\left(D_{t t}+D_{x x x x}+b(x)\right)^{-1}\left(1+\lambda\left(-b(x) u^{-}+\epsilon h(x, t)\right)\right), B_{\eta}(y), 0\right) \\
& =d_{L S}\left(u-\left(D_{t t}+D_{x x x x}+b(x)\right)^{-1}(1), B_{\eta}(y), 0\right) \\
& =d_{L S}\left(u-\left(D_{t t}+D_{x x x x}\right)^{-1}(1-b(x) u), B_{\eta}(y), 0\right) .
\end{aligned}
$$

The equation $\left(D_{t t}+D_{x x x x}\right) u=-b(x) u+1$ has a unique solution $u(x, t)=$ $y(x)$. Thus

$$
\begin{aligned}
& d_{L S}\left(u-\left(D_{t t}+D_{x x x x}\right)^{-1}(1-b(x) u), B_{\eta}(y), 0\right) \\
& =d_{L S}\left(u+\left(D_{t t}+D_{x x x x}\right)^{-1}(b(x) u), B_{\eta}(0), 0\right) .
\end{aligned}
$$


The eigenvalues $\rho$ of the operator $u+\left(D_{t t}+D_{x x x x}\right)^{-1}(b(x) u)$ are connected with the eigenvalues $\mu_{n}^{ \pm}$of $D_{t t}+D_{x x x x}$ by

$$
u+\left(D_{t t}+D_{x x x x}\right)^{-1}(b(x) u)=\rho u o r u_{t t}+u_{x x x x}=\frac{b(x)}{\rho-1} u
$$

or $\rho=1+\frac{b(x)}{\mu}$. It follows from (1.9) that there is just one negative eigenvalue which correspond to $\lambda_{10}=-3$. Thus the usual method of approximating on finite-dimensional subspaces spanned by eigenvectors with dimension going to infinity shows that the desired degree is -1 . Thus we prove the lemma.

\section{Proof of Theorem 1.1}

By Lemma 2.4, there exists a large number $R>0$ (depending on $C$ ) such that the Leray-Schauder degree

$$
d_{L S}\left(u-\left(D_{t t}+D_{x x x x}\right)^{-1}\left(-b(x) u^{+}+1+\epsilon h+s \psi_{0}(x)\right), B_{R}(0), 0\right)=0
$$

for $R>C$ and $s \leq s_{*}$. By Lemma 2.7, there exists a constant $\eta>0$, $\epsilon_{0}>0$ such that the Leray-Schauder degree

$$
d_{L S}\left(u-\left(D_{t t}+D_{x x x x}\right)^{-1}\left(-b(x) u^{+}+1+\epsilon h\right), B_{\eta}(y), 0\right)=-1
$$

for $|\epsilon|<\epsilon_{0}$, where $y$ is the positive solution of (2.12). The LeraySchauder degree in the region $B_{R}(0) \backslash B_{\eta}(y)$ is 1 , so there exists the second solution of (1.1) in the region $B_{R}(0) \backslash B_{\eta}(y)$. Therefore there exist at least two solutions of (1.1). Thus we complete the proof.

\section{References}

[1] Q. H. Choi and T. Jung, The study of a nonlinear suspension bridge equation by a variational reduction method, Appl. Anal. 50(1993), 73-92.

[2] G. Fournier, D. Lupo, M. Ramos and M. Willem, Limit relative category and critical point theory, Dynam. Report, 3(1993), 1-23.

[3] P. J. McKenna and W. Walter, Nonlinear oscillations in a suspension bridge, Arch. Ration. Mech. Anal. 98(2)(1987), 167-177.

[4] A. M. Micheletti and C. Saccon, Multiple nontrivial solutions for a floating beam equation via critical point theory, J. Differential Equations, 170(2001), 157-179. 
Department of Mathematics

Kunsan National University

Kunsan 573-701, Korea

E-mail: tsjung@kunsan.ac.kr

Department of Mathematics Education

Inha University

Incheon 402-751, Korea

E-mail: qheung@inha.ac.kr 